\title{
O método dos Tableaux Aplicado ao Cálculo Trivalente e Intuicionista I1
}

\author{
E. O. V. SANTOS ${ }^{1}$ e L. H. C. SILVESTRINI ${ }^{2 *}$ \\ Recebido em 15 de fevereiro de 2020 / Aceito em 15 de fevereiro de 2021
}

\begin{abstract}
RESUMO. O cálculo I1 foi introduzido em 1995 por Sette e Carnielli. Este sistema possui um caráter intuicionista, no mesmo sentido do sistema lógico desenvolvido por Arend Heyting (1898-1980), o qual surgiu como a lógica subjacente a Matemática Intuicionista, ou construtivista, por exemplo, $\neg \neg A \rightarrow A$ não ser uma tautologia em I1. Ademais, o cálculo I1 é uma lógica trivalorada que, ao contrário da lógica clássica, não admite apenas dois valores de verdade, mas sim três, estes são $\mathrm{T}, \mathrm{F}^{*} \mathrm{e}$ F. Os valores $\mathrm{T}$ e $\mathrm{F}$ denotam, respectivamente, verdade e falsidade, enquanto que $\mathrm{F}^{*}$ pode ser interpretado como "falsidade por falta de evidência positiva". O ambiente semântico dessa lógica, há apenas um valor distinguido. Neste trabalho, desenvolvemos um método dedutivo alternativo ao axiomático para o sistema I1, ou seja, introduzimos um sistema de tableaux analíticos para tal lógica. Estabelecemos, por meio de teoremas, que toda dedução obtida do sistema axiomático também será deduzida pelo sistema de tableaux proposto.
\end{abstract}

Palavras-chave: lógica intuicionista, lógica trivalente, método dos tableaux analíticos.

\section{INTRODUÇÃO}

A lógica como ciência surgiu na Antiguidade. Entre os gregos, diversas escolas produziram trabalhos sobre lógica. Porém, foi Aristóteles (384 - 322 a.C.), filósofo grego, quem apresentou de maneira mais elaborada os primeiros textos de lógica e explicitou princípios que caracterizam o que denominamos de lógica aristotélica.

A lógica clássica é compreendida pelo cálculo de predicados de primeira ordem com identidade e símbolos funcionais, com características a obediência aos princípios lógicos clássicos. Há inúmeras aplicações para a lógica clássica, porém, em alguns casos ela não é adequada. E para esses casos surgiram as lógicas não-clássicas, como a I1, a qual será abordada nesta pesquisa.

A lógica intuicionista, segundo Mortari [6], é a lógica da matemática intuicionista, e o intuicionismo, uma corrente dentro da matemática originada por L. E. J. Brouwer (1881-1966). Assim sendo, um intuicionista só aceita demonstrações de existência de objetos matemáticos por

\footnotetext{
*Autor correspondente: Luiz Henrique da Cruz Silvestrini - E-mail: 1h.silvestrini@ unesp.br

${ }^{1}$ Faculdade de Ciências, Universidade Estadual Paulista - UNESP, Av. Eng. Luiz Edmundo Carrijo Coube, 14-01, 17033360, Bauru, SP, Brasil - E-mail: elias.ov.santos@ unesp.br http://orcid.org/0000-0001-5205-640X

2 Departamento de Matemática, Faculdade de Ciências - UNESP, Universidade Estadual Paulista, Av. Eng. Luiz Edmundo Carrijo Coube, 14-01, 17033-360, Bauru, SP, Brasil - E-mail: lh.silvestrini@ unesp.br https://orcid.org/0000-0002-00649753
} 
construção, não aceitando demonstrações por redução ao absurdo, dedução indireta muito usada na Matemática Clássica, em que se demonstra que muitos entes existem porque supor o contrário implicaria em uma contradição. Assim, uma das propriedades que a lógica intuicionista rejeita é o princípio da dupla negação: $\neg \neg A \rightarrow A$ não é válida intuicionisticamente.

Para Carnielli e Lima-Marques [1], uma lógica é dita polivalente (multivalorada) se admite mais de dois valores de verdade, como consequência, tal lógica derroga o princípio clássico da bivalência.

A lógica I1, uma lógica de caráter intuicionista e trivalente, apresenta além dos valores de verdade tradicionais $\mathrm{T}$ e F, o valor de verdade $\mathrm{F}^{*}$, que pode ser interpretado como "falsidade por falta de evidência positiva”. No ambiente semântico desta lógica, somente o valor T é distinguido. Este sistema foi introduzido por Sette e Carnielli [7] e possui um caráter intuicionista no sentido de, por exemplo, $\neg \neg A \rightarrow A$ não ser uma tautologia em I1.

Os tableaux, também conhecidos como árvores de refutação, são um método de dedução indireta, ou redução ao absurdo no sentido metateórico, utilizados para verificar a validade de um argumento. Um tableau clássico também pode ser definido como uma árvore ordenada diádica, segundo Smullyan [10]. Adotamos, neste trabalho, o termo original do francês tableaux, em que há duas formas de escrita, a saber, tableau e tableaux, empregadas, respectivamente, no singular e plural.

O objetivo central deste artigo será introduzir o sistema I1 através do método dedutivo por tableaux analíticos. Para isso, serão explicitadas as cláusulas de fechamento e regras de expansão do nosso tableau TI1; em seguida, provaremos a equivalência dedutiva da lógica I1 com nosso sistema de tableaux TI1.

\section{LÓGICA INTUICIONISTA E TRIVALENTE I1}

A lógica I1, apresentada por Sette e Carnielli [7], foi criada a partir da Semântica de Sociedades, a qual, segundo Silvestrini [9], é um tipo de construção lógica, que foi introduzida, em 1999, por Carnielli e Lima-Marques [1] e permite obter novas lógicas a partir da combinação dos agentes, i.e., das valorações de uma lógica já existente. Esta abordagem situa-se numa área de estudo relativamente nova dentro da lógica, a qual estuda combinações entre diferentes sistemas lógicos.

Segundo Fernández [3], a Semântica de Sociedades foi proposta também como uma tentativa para dar uma nova resposta à questão, sempre presente dentro da lógica, de expressar adequadamente casos que possam permitir a presença de enunciados contraditórios.

De acordo com Loparic e da Costa [5], "Um sistema lógico é paracompleto se pode funcionar como lógica subjacente de teorias nas quais existem fórmulas fechadas tais que essas fórmulas e suas negações sejam simultaneamente falsas. Nós chamamos tais teorias paracompletas”. 
A partir disto, Queiroz [2] conclui que "Uma grande classe de lógicas é paracompleta, entre elas a lógica intuicionista. Neste sentido, adotaremos o termo paracompleta para indicar as lógicas que não satisfazem o terceiro excluído".

Assim, temos a lógica I1 como uma lógica paracompleta, trivalente e intuicionista, que apresenta além dos valores de verdade clássicos $\mathrm{T}$ e $\mathrm{F}$, o valor de verdade $\mathrm{F}^{*}$, que pode ser interpretado como "falsidade por falta de evidência positiva". No ambiente semântico desta lógica, somente o valor T é distinguido. No ambiente matricial, trabalharemos com valorações $1, \frac{1}{2}$ e 0 , para T, $\mathrm{F}^{*}$ e F, respectivamente.

O sistema I1 possui um caráter intuicionista no sentido de, por exemplo, a fórmula $\neg \neg A \rightarrow A$ não ser, ao menos no nível atômico, uma tautologia em I1. Neste sistema todos os axiomas do bem conhecido sistema de Heyting para a lógica intuicionista são válidos, e a lei do terceiro excluído não é válida a partir da definição de disjunção daquele sistema. I1 pode ser caracterizada, axiomaticamente, como veremos a seguir.

\subsection{Sintaxe para a lógica I1}

Introduziremos a sintaxe, segundo Carnielli e Lima-Marques [1].

\section{Esquemas de Axiomas:}

1. $A \rightarrow(B \rightarrow A)$

2. $(A \rightarrow(B \rightarrow C)) \rightarrow((A \rightarrow B) \rightarrow(A \rightarrow C))$

3. $(\neg \neg A \rightarrow \neg B) \rightarrow((\neg \neg A \rightarrow B) \rightarrow \neg A)$

4. $\neg \neg(A \rightarrow B) \rightarrow(A \rightarrow B)$

E a Modus Ponens $(A, A \rightarrow B \vdash B)$ é a única regra de inferência do sistema.

\subsection{Semântica da lógica I1}

A lógica I1 apresenta os conectivos primitivos $\rightarrow \mathrm{e} \neg$. Assim, podemos definir a expressão matricial desta lógica como I1 $=\left\langle\left\{1, \frac{1}{2}, 0\right\},\{\rightarrow, \neg\},\{1\}\right\rangle$, em que os valores de verdade são $1, \frac{1}{2}, 0$, dos quais apenas o valor 1 é distinguido. Intuitivamente, "1" e "0" denotam, respectivamente, verdade e falsidade, enquanto " $\frac{1}{2}$ " pode ser entendido como "falsidade por falta de evidência positiva".

Assim, seguem as interpretações em matrizes desses operadores básicos da I1. Lembramos que, de forma usual, o operador binário $A \rightarrow B$ adota $A$ como um elemento da primeira coluna à esquerda e $B$ como um elemento da primeira linha do topo da tabela: 


\begin{tabular}{|c||c|c|c|}
\hline & 1 & $\frac{1}{2}$ & 0 \\
\hline \hline$\neg$ & 0 & 0 & 1 \\
\hline
\end{tabular}

\begin{tabular}{|c||c|c|c|}
\hline$\rightarrow$ & 1 & $\frac{1}{2}$ & 0 \\
\hline \hline 1 & 1 & 0 & 0 \\
\hline$\frac{1}{2}$ & 1 & 1 & 1 \\
\hline 0 & 1 & 1 & 1 \\
\hline
\end{tabular}

Segundo Carnielli e Lima-Marques [1], I1 pode ser visto como um subsistema do cálculo proposicional clássico e é maximal, no sentido de que ao adicionarmos aos seus axiomas qualquer tautologia clássica que não seja uma I1-tautologia, o sistema resultante colapsa com o cálculo proposicional clássico. Além disso, é possível definir em I1 uma negação fraca $\sim A=_{\text {def }} A \rightarrow \neg A$, a qual apresenta todas as propriedades da negação clássica, conforme a tabela:

\begin{tabular}{|c||c|c|c|}
\hline & 1 & $\frac{1}{2}$ & 0 \\
\hline \hline$\tilde{ᄀ}$ & 0 & 1 & 1 \\
\hline
\end{tabular}

E pode ser definida a conjunção $A \wedge B$ e a disjunção $A \vee B$ para o sistema como se segue:

$$
\begin{aligned}
& A \wedge B=\operatorname{def} \neg(A \rightarrow \tilde{\neg} B) \\
& A \vee B={ }_{\operatorname{def}} \neg(\tilde{\neg} A \rightarrow B)
\end{aligned}
$$

\begin{tabular}{|c||c|c|c|}
\hline$\wedge$ & 1 & $\frac{1}{2}$ & 0 \\
\hline \hline 1 & 1 & 0 & 0 \\
\hline$\frac{1}{2}$ & 0 & 0 & 0 \\
\hline 0 & 0 & 0 & 0 \\
\hline
\end{tabular}

\begin{tabular}{|c||c|c|c|}
\hline$\vee$ & 1 & $\frac{1}{2}$ & 0 \\
\hline \hline 1 & 1 & 1 & 1 \\
\hline$\frac{1}{2}$ & 1 & 0 & 0 \\
\hline 0 & 1 & 0 & 0 \\
\hline
\end{tabular}

A partir desta semântica, é demonstrada a adequação, i.e., os metateoremas de correção e completude, acerca do sistema axiomático I1. Tal adequação foi apresentada por Sette e Carnielli [7].

\section{TABLEAUX COMO ALTERNATIVA AO SISTEMA AXIOMÁTICO}

Abordaremos o método dos tableaux analíticos, explicitando inicialmente suas origens. Apresentamos o método de tableaux do cálculo proposicional clássico, sendo estes necessários para ponto de partida de criação do sistema de tableaux que desenvolvemos para a lógica I1, que apresentaremos no capítulo seguinte.

\subsection{O método dos tableaux analíticos}

O método dos tableaux analíticos é um método de prova baseado em refutação que permite verificar se uma determinada fórmula é ou não um teorema de uma teoria, introduzido por Raymond M. Smullyan (1919-2017), matemático e lógico estadunidense, em 1968 [10]. Segundo Fitting [4] a história dos tableaux começa com Gerhard Gentzen (1909-1945), matemático e lógico alemão, e culmina com Smullyan. 
Os sistemas de provas de Gentzen eram caracterizados por admitirem o princípio da subfórmula, isto é, se uma fórmula A é demonstrável, então A tem uma demonstração em que ocorrem apenas subfórmulas de A. Todos os trabalhos subsequentes que levaram ao desenvolvimento dos sistemas de tableaux foram de algum modo, inspirados pelos trabalhos de Gentzen em relação aos sistemas de provas.

O trabalho de Gentzen foi desenvolvido posteriormente por Evert Willem Beth (1908-1964), filósofo e lógico holandês, que apresentou o método dos tableaux semânticos, que também utiliza o princípio da subfórmula. Além disso, a proposta de Smullyan pode ser considerada uma variante dos métodos de prova de Hintikka (1929-2015).

O método de tableaux analíticos é um sistema de prova automática de teoremas, caracterizado como um algoritmo. Por isso, é um sistema de decisão para as fórmulas válidas de uma determinada lógica, do mesmo modo que as tabelas de verdade são para a lógica proposicional clássica. A principal característica dos sistemas de tableaux é que ele é um método de refutação, ou seja, para demonstrar que alguma fórmula A é válida, começamos supondo que ela não é, e derivamos as consequências dessa suposição. Se isso nos levar a um absurdo, metateórico, a suposição inicial é falsa, logo concluímos que A é verdadeira. Caso contrário, A é falsa e o tableau nos mostra imediatamente um contra-exemplo.

Na próxima seção, apresentamos o método de tableaux analíticos para o cálculo proposicional clássico, segundo Smullyan [10].

\subsection{Tableaux analíticos para o cálculo proposicional clássico}

Nesta seção, apresentaremos o método de tableaux analíticos para o cálculo proposicional clássico, baseado no trabalho de Smullyan [10], o qual define um tableau analítico por meio do uso das árvores ordenadas diádicas. Assim, assumiremos os conceitos clássicos à lá Smullyan.

Definição 3.1 (Tableau analítico). Um tableau analítico é uma árvore ordenada diádica T, cujos pontos são fórmulas. Um tableau T para uma fórmula $\varphi$ qualquer é construído do seguinte modo: Iniciamos o tableau com $\neg \varphi$ em sua origem (raiz da árvore) e a construção ou expansão dos ramos dá-se pela aplicação de uma das regras de expansão.

As regras de expansão para o cálculo proposicional clássico, de acordo com Smullyan [10, p. 20], podem ser de dois tipos. As fórmulas do tipo $\alpha$, a expansão do ramo dá-se sem ocorrer bifurcação. As fórmulas do tipo $\beta$, a expansão do ramo dá-se pela ocorrência de bifurcação.

Um ramo de um tableau é denominado um ramo fechado quando existem neste ramo pontos que correspondam às fórmulas $\delta \mathrm{e} \neg \delta$. Quando um ramo apresentar uma fórmula e sua negação como pontos distintos, utilizaremos ' $\times$ ' para simbolizar que o ramo é fechado. Um tableau para uma fórmula dada $\varphi$ é fechado quando todos os ramos são fechados.

Dizemos que $\Gamma$ é um conjunto fechado de fórmulas quando é possível a construção de um tableau fechado para a conjunção finita de fórmulas de $\Gamma$; caso contrário, dizemos que $\Gamma$ é um conjunto 
aberto de fórmulas. Uma fórmula $\varphi$ é consequência analítica ou gerada de um conjunto $\Gamma$ de fórmulas, o que denotaremos por $\Gamma \Vdash \varphi$, quando $\Gamma \cup\{\neg \varphi\}$ for um conjunto fechado de fórmulas. Uma fórmula $\varphi$ é demonstrável em $\mathrm{T}$, o que denotamos por $\Vdash \varphi$, se é possível construir um tableau fechado a partir da fórmula inicial $\neg \varphi$, ou seja, o conjunto $\{\neg \varphi\}$ é fechado.

Um ramo é completo quando nenhuma regra de expansão pode ser aplicada em qualquer um dos pontos deste ramo, ou quando o ramo é fechado. Um tableau é completo se todos os seus ramos são completos.

\section{UM SISTEMA DE TABLEAUX PARA A LÓGICA I1}

Neste capítulo, introduziremos o método dos tableaux analíticos para a lógica I1, originalmente apresentada por Sette e Carnielli [7] em um sistema axiomático.

Desta maneira, apresentaremos, de modo original, as regras de expansão para a TI1, as quais foram obtidas a partir da análise das matrizes trivalentes da lógica I1. Também abordaremos os quatro tipos de categorias em que elas se enquadram e mostraremos serem dedutivamente equivalentes com a versão axiomática de I1.

\subsection{Tableaux para a Lógica I1}

O método dos tableaux introduzido por Smullyan [10], apresentado no capítulo anterior, foi desenvolvido para a lógica proposicional clássica e para a lógica de primeira ordem clássica. Contudo a lógica I1 é intuicionista e trivalente. Diante disso, as regras de expansão apresentadas por Smullyan não abrangem os aspectos não-clássicos da I1. Logo, faz-se necessário regras de expansão específicas para o desenvolvimento dos tableaux para a lógica I1.

Assim, apresentamos um sistema de tableaux analíticos para a lógica I1, o qual denotamos por TI1.

As regras de expansão utilizadas no sistema TI1 são motivadas pelas regras da lógica clássica, para as fórmulas de comportamento clássico, acrescidas de aspectos específicos para as fórmulas da lógica I1.

Classificamos as regras de expansão para TI1 em quatro tipos, com cada fórmula gerando de um a quatro ramos, como segue:

- Fórmulas do tipo $\alpha$ : são fórmulas em que suas consequências são diretas e não temos ramificações. As consequências permanecem no mesmo ramo.

- Fórmulas do tipo $\beta$ : neste caso, as consequências bifurcam em dois ramos, gerando dois ramos distintos.

- Fórmulas do tipo $\gamma$ : são fórmulas com consequências indiretas e ramificações em três ramos distintos. 
- Fórmulas do tipo $\delta$ : são fórmulas com consequências indiretas e ramificações em quatros ramos distintos.

Assim, cada uma das regras de expansão de TI1 está em uma destas quatro categorias apresentadas, ou seja, temos as seguintes possibilidades de expansão do tableau: ou não há ramificações, ou ocorre uma ramificação em dois ramos distintos, ou ramificam em três ramos diferentes, ou ramificam em quatro ramos diferentes.

Aqui, há uma diferença com o tableau clássico, pois no ambiente clássico, temos como consequências da fórmula inicial, no máximo dois ramos distintos, lembrando que tanto a lógica proposicional clássica, como a de primeira ordem clássica são lógicas com apenas duas valorações possíveis, a saber, 0 para falso e 1 para verdadeiro. Para os tableaux no ambiente da lógica trivalente I1, temos fórmulas que podem gerar até quatro ramos distintos.

\subsection{Cláusulas de Fechamento TI1}

Definição 4.2 (Ramo fechado em TI1). Um ramo no sistema TIl é fechado quando uma mesma fórmula $\lambda$ possui valores distintos neste mesmo ramo, ou seja, quando uma das três seguintes situações ocorrer:

i) $1 \lambda e \frac{1}{2} \lambda$

ii) $1 \lambda$ e $0 \lambda$ (cláusula clássica);

iii) $\frac{1}{2} \lambda e 0 \lambda$.

Ou ainda, quando ocorrer o seguinte caso:

iv) encontramos uma fórmula $\alpha$, sendo $\alpha$ uma proposição composta formada pelos conectivos $\neg, \rightarrow, \wedge$ ou $\vee$, com valor $\frac{1}{2}$.

Observação 1. Simbolizamos o ramo fechado colocando o símbolo ' $\times$ ' no final do ramo, seguido das informações $(a, b)$ para os casos (i), (ii) e (iii), onde a e b são as linhas que ocorreram os valores distintos para o fechamento do tableau. Ou ainda, o símbolo ' $\times$ ' seguido de qual conectivo com valor $\frac{1}{2}$ foi o responsável pelo fechamento do ramo, pois temos $\left[\frac{1}{2} \neg\right],\left[\frac{1}{2} \rightarrow\right],\left[\frac{1}{2} \wedge\right]$ $e\left[\frac{1}{2} \vee\right]$ como Cláusulas de Fechamento pelo caso (iv).

\subsection{Regras de Expansão TI1}

Estando definidas as cláusulas de fechamento de nosso sistema TI1, introduziremos as regras de expansão para esta lógica, as quais estão classificadas de acordos com seus respectivos operadores: 
Negação
$[0 \neg] \quad 0 \neg A$
$[1 \neg] \quad 1 \neg A$
$1 A \quad \frac{1}{2} A$
$0 A$

\section{Condicional}

$\begin{array}{ccccc}{[0 \rightarrow]} & 0(A \rightarrow B) & {[1 \rightarrow]} & 1(A \rightarrow B) \\ & - & & & \\ 1 A & 1 A & 0 A & \frac{1}{2} A & 1 B \\ \mid & \mid & & & \end{array}$

\section{Conjunção}

\begin{tabular}{|c|c|c|}
\hline$[0 \wedge]$ & $0(A \wedge B)$ & \\
\hline & - & \\
\hline $0 A$ & $\frac{1}{2} A \quad 0 B$ & $\frac{1}{2} B$ \\
\hline
\end{tabular}

\section{Disjunção}

\begin{tabular}{|c|c|}
\hline$[0 \vee]$ & $\begin{array}{c}0(A \vee B) \\
-\end{array}$ \\
\hline $0 A$ & $\frac{1}{2} A \quad 0 A$ \\
\hline$\frac{1}{2} B$ & $\frac{1}{2} B \quad 0 B$ \\
\hline
\end{tabular}

$[1 \vee] \quad 1(A \vee B)$

$1 A \quad 1 B$

As regras de expansão apresentadas foram obtidas por meio da análise das matrizes trivalentes da lógica I1, introduzidas por Carnielli e Lima-Marques [1].

Com relação ao tipo de fórmulas no tableau, as regras de expansão de TI1 podem ser classificadas:

- Tipo $\alpha:[1 \neg],[1 \wedge]$.

- Tipo $\beta:[0 \neg],[0 \rightarrow],[1 \vee]$.

- Tipo $\gamma:[1 \rightarrow]$.

- Tipo $\delta:[0 \wedge],[0 \vee]$. 
Verificamos então, que podemos gerar até quatro ramificações, mas, de um modo geral, seguimos a intuição dos resultados do tableaux para lógica clássica.

Como não temos a valoração $\frac{1}{2}$ para nenhum operador nas matrizes lógica $\mathrm{I} 1$, definidas por Carnielli e Lima-Marques [1], então não temos regras de expansão para as mesmas, uma vez que quando ocorrer esta situação no tableau, fechamos imediatamente o ramo. Por isso definimos tal situação como cláusula de fechamento, conforme Definição 4.2, item (iv).

\subsection{Equivalência dedutiva entre TI1 e I1}

Após a criação das cláusulas de fechamento e regras de expansão do nosso sistema TI1, o objetivo central de nossa pesquisa é demonstrar a equivalência entre o sistema axiomático I1 e o sistema de tableaux TI1. Esta tarefa será contemplada por meio dos seguintes (meta)teoremas, que demonstraremos nas próximas seções.

Etapa 1 [Teorema da Corretude Analítica]

$$
\text { Se } \Delta \vdash \varphi \text {, então } \Delta \Vdash \varphi
$$

A Demonstração da Corretude Analítica será dada no Teorema 4.1

Etapa 2 [Teorema da Completude Analítica]

$$
\operatorname{Se} \Delta \Vdash \varphi \text {, então } \Delta \vDash \varphi
$$

A Demonstração da Completude Analítica será dada no Teorema 4.2

Em que: $\Delta \Vdash \varphi$ denota que a fórmula $\varphi$ é consequência analítica de um conjunto $\Delta$ de fórmulas, deduzidas em nosso TI1. No sistema axiomático da lógica I1, denotamos $\varphi$ uma consequência lógica (sintática) de $\Delta$, por $\Delta \vdash \varphi$, e, $\varphi$ uma consequência semântica de $\Delta$, por $\Delta \vDash \varphi$. A correção e completude usuais, ou seja, $\Gamma \vdash \varphi \Leftrightarrow \Gamma \vDash \varphi$ estão demonstradas em Sette e Carnielli [7].

\subsection{Da dedução axiomática para a dedução em tableaux}

Neste momento, apresentaremos inicialmente como será feito a adequação, isto é, como provaremos a equivalência dedutiva entre o sistema axiomático I1 e nosso sistema de tabelaux TI1. Assim, para demonstrarmos que cada dedução obtida na lógica I1 também é deduzida em nosso sistema TI1 e vice-versa, provaremos o seguinte:

$$
\Gamma \Vdash \varphi \Leftrightarrow \Gamma \vdash \varphi \Leftrightarrow \Gamma \vDash \varphi
$$


Como o sistema axiomático da lógica I1 tem a equivalência semântica demonstrada por meio das provas de corretude e completude apresentadas em Sette e Carnielli [7], sabemos que:

$$
\Gamma \vdash \varphi \Leftrightarrow \Gamma \vDash \varphi
$$

Seguiremos, então, o seguinte caminho:

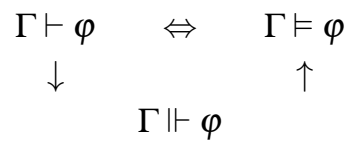

A seta para baixo e a seta para cima do diagrama anterior, mostram as passagens originais de nosso trabalho.

Teorema 4.1. Se $\Delta \vdash \varphi$, então $\Delta \Vdash \varphi$

Demonstração. Seja $\delta_{1}, \ldots, \delta_{n}\left(\delta_{n}=\varphi\right)$ a dedução de $\varphi$ a partir de $\Delta$.

Demonstraremos por indução sobre o comprimento (n) da dedução a partir de $\Delta$, que se $\Delta \vdash \varphi$, então $\Delta \Vdash \varphi$.

Base de indução: a dedução de $\varphi$ a partir de $\Delta$ tem uma linha de demonstração, isto é, $\delta_{1}=\varphi$. Deste modo, temos duas possibilidades:

(a) $\varphi$ é uma premissa, ou seja, $\delta_{1} \in \Delta$, ou

(b) $\varphi$ é um axioma da teoria I1.

No caso (a), se $\varphi \in \Delta$, conseguimos provar que $\Delta \Vdash \varphi$, já que, por hipótese, supomos $\Delta=\Gamma \cup\{\varphi\}$ e, assim, $\Gamma \cup\{\varphi\} \cup\{\neg \varphi\}$ é um conjunto fechado de fórmulas, e isto mostra que $\varphi$ é consequência analítica de $\Delta$, ou seja, $\Gamma \cup\{\varphi\} \Vdash \varphi$.

Para o caso (b), é necessário avaliarmos os axiomas presentes no sistema I1. Do mesmo modo feito no item anterior, para demonstrarmos $\Delta \Vdash \varphi$, devemos provar que $\varphi$ é uma consequência analítica de $\Delta$, isto; é, $\Delta \cup\{\neg \varphi\}$ é fechado. Assim, estaremos mostrando que $\neg(\Delta \rightarrow \varphi)$ é inconsistente, ou seja, ao negarmos que o conjunto de premissas implica logicamente em $\varphi$, geramos um tableau fechado.

Avaliemos agora, quando $\delta_{1}$ for um axioma para o sistema I1. Por alguns tableaux serem demasiadamente longos, abreviaremos o desenvolvimento destes para apresentação neste artigo por motivo de espaço.

i) Seja $\delta_{1}$ uma instância do esquema de axioma $A x_{1}$, isto é:

$\delta_{1} \equiv A \rightarrow(B \rightarrow A)$

Geramos um tableau fechado para $0 \delta_{1}$ e $\frac{1}{2} \delta_{1}$ (ou seja, $\Delta \cup\left\{\neg \delta_{1}\right\}$ é fechado, então $\delta_{1}$ é consequência analítica) como segue: 
- Para $\frac{1}{2} \delta_{1}$ temos o tableau:

$$
\begin{aligned}
& \Delta \\
\frac{1}{2}(A \rightarrow & (B \rightarrow A)) \quad \text { raiz } \\
& \mid \\
& \times \\
{\left[\frac{1}{2} \rightarrow\right] \text { em } 0 } &
\end{aligned}
$$

- E para $0 \delta_{1}$ temos o tableau:

$$
\begin{aligned}
& 0 \quad 0(A \rightarrow(B \rightarrow A)) \quad \text { raiz } \\
& \begin{array}{cccc}
1 & 1 A & 1 A & {[0 \rightarrow] \text { em } 0}
\end{array} \\
& 2 \quad \frac{1}{2}(B \rightarrow A) \quad 0(B \rightarrow A) \quad[0 \rightarrow] \text { em } 0
\end{aligned}
$$

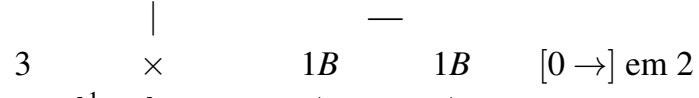

$$
\begin{aligned}
& \text { (1 e 4) (1 e 4) }
\end{aligned}
$$

ii) Seja $\delta_{1} \in A x_{2}$, ou seja, temos uma instância de $A x_{2}$ :

$$
\delta_{1} \equiv(A \rightarrow(B \rightarrow C)) \rightarrow((A \rightarrow B) \rightarrow(A \rightarrow C))
$$

Assim, temos um tableau fechado para $0 \delta_{1}$ e $\frac{1}{2} \delta_{1}$ :

- Para $\frac{1}{2} \delta_{1}$ temos o tableau:

$$
\begin{aligned}
0 \quad \frac{1}{2}((A \rightarrow(B \rightarrow C)) \rightarrow & ((A \rightarrow B) \rightarrow(A \rightarrow C))) \quad \text { raiz } \\
& \mid \\
& \times \\
{\left[\frac{1}{2} \rightarrow\right] \text { em } 0 } &
\end{aligned}
$$

- E para $0 \delta_{1}$ temos o tableau, o qual alguns ramos foram omitidos por conta de espaço: 


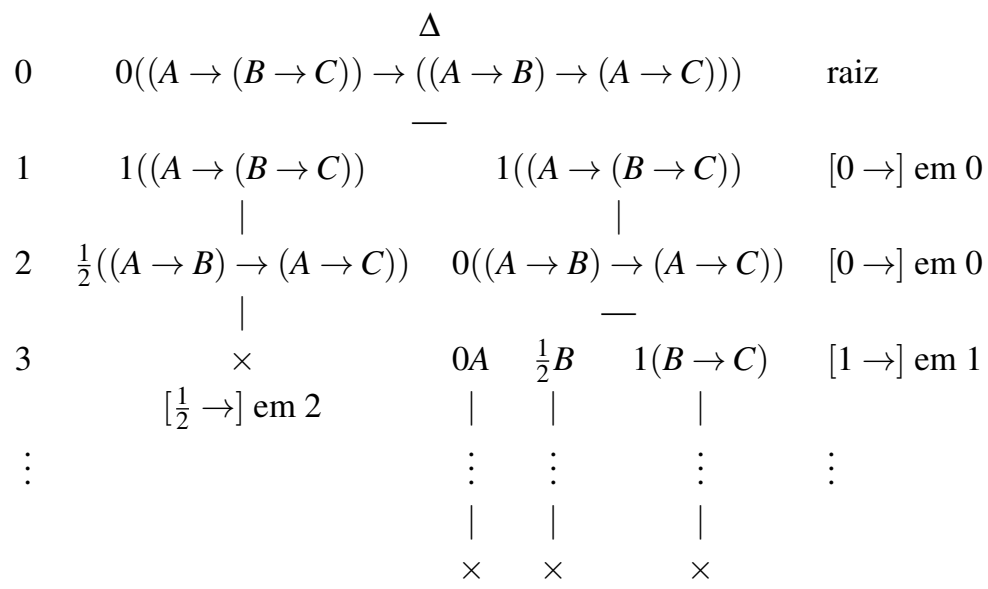

iii) $\delta_{1}$ é uma instância de $A x_{3}$.

$$
\delta_{1} \equiv(\neg \neg A \rightarrow \neg B) \rightarrow((\neg \neg A \rightarrow B) \rightarrow \neg A)
$$

Temos um tableau fechado para $0 \delta_{1}$ e $\frac{1}{2} \delta_{1}$ :

- Para $\frac{1}{2} \delta_{1}$ temos o tableau:

$$
\begin{aligned}
0 \quad \frac{1}{2}((\neg \neg A \rightarrow \neg B) \rightarrow & ((\neg \neg A \rightarrow B) \rightarrow \neg A)) \quad \text { raiz } \\
& \mid \\
& \times \\
{\left[\frac{1}{2} \rightarrow\right] \text { em } 0 } &
\end{aligned}
$$

- E para $0 \delta_{1}$ temos o tableau, o qual alguns ramos omitidos por conta de espaço:

$\Delta$

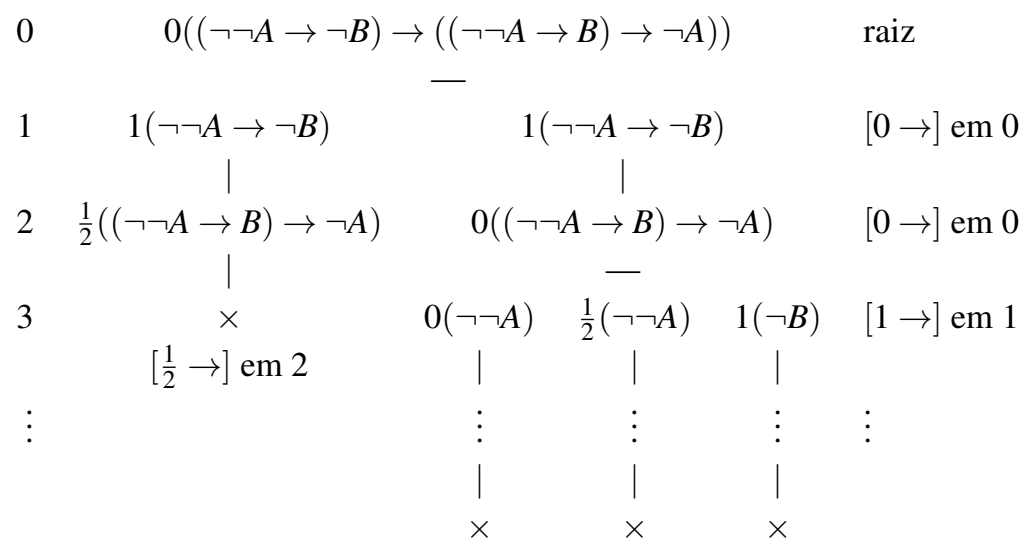


iv) $\delta_{1}$ é uma instância de $A x_{4}$.

$$
\delta_{1} \equiv \neg \neg(A \rightarrow B) \rightarrow(A \rightarrow B)
$$

Temos o tableau fechado para $0 \delta_{1}$ e $\frac{1}{2} \delta_{1}$ :

- Para $\frac{1}{2} \delta_{1}$ temos o tableau:

$$
\begin{aligned}
& \Delta \\
& 0 \quad \frac{1}{2}(\neg \neg(A \rightarrow B) \rightarrow(A \rightarrow B)) \quad \text { raiz } \\
& \times \\
& {\left[\frac{1}{2} \rightarrow\right] \text { em } 0}
\end{aligned}
$$

- E para $0 \delta_{1}$ temos o tableau:

$$
\begin{aligned}
& \Delta \\
& 0 \\
& 0(\neg \neg(A \rightarrow B) \rightarrow(A \rightarrow B)) \quad \text { raiz } \\
& 1 \quad 1(\neg \neg(A \rightarrow B)) \\
& 1(\neg \neg(A \rightarrow B)) \\
& {[0 \rightarrow] \text { em } 0} \\
& 2 \quad \frac{1}{2}(A \rightarrow B) \\
& 0(A \rightarrow B) \\
& {[0 \rightarrow] \text { em } 0} \\
& 3 \\
& 0(\neg(A \rightarrow B)) \\
& {[1 \rightarrow] \text { em } 1} \\
& {\left[\frac{1}{2} \rightarrow\right] \text { em } 2} \\
& 4 \\
& \begin{array}{cc}
1(A \rightarrow B) & \frac{1}{2}(A \rightarrow B) \\
\mid & \mid \\
\times & \times
\end{array} \\
& (2 \text { e } 4) \quad\left[\frac{1}{2} \rightarrow\right] \text { em } 4
\end{aligned}
$$

Hipótese de indução: A dedução de $\varphi$ a partir de $\Delta$ em $\Delta \vdash \varphi$, tem comprimento $n(n>1)$, e o resultado do teorema vale para toda fórmula que pode ser deduzida a partir de $\Delta$ e que possua o comprimento de sua dedução menor que o comprimento $n$.

(Passo Indutivo) Devemos verificar que o teorema vale para $\varphi=\delta_{n}$.

Temos as seguintes possibilidades para $\delta_{n}$ ter sido originado num passo $\mathrm{n}$ da dedução.

- $\delta_{n}=\varphi$ é um axioma de I1.

Pelo resultado da cláusula (b) da base, temos que $\Delta \Vdash \varphi$.

- $\varphi \in \Gamma$.

Obtemos $\Delta \Vdash \varphi$ do mesmo modo que foi feito na cláusula (a) da base de indução. 
- Se a dedução de $\delta_{n}=\varphi$ advém por meio da regra de inferência, então necessitamos verificar se a regras é de fato válida no sistema TI1.

- Por Modus Ponens: Se $\delta_{n}$ é deduzida por esta regra, então podemos estabelecer duas outras fórmulas $\delta_{i}$ e $\delta_{j}$ na dedução que satisfazem a seguinte identidade:

$$
\delta_{j}=\delta_{i} \rightarrow \delta_{n}, \text { para algum } i \neq j \text { e } i, j<n .
$$

Então, pela hipótese de indução, podemos dizer que:

(h1.1): $\Gamma \cup\left\{0 \delta_{i}\right\}$ é fechado, pois $\delta_{i}$ possui comprimento menor que n, e então $\Gamma \Vdash \delta_{i}$.

(h1.2): $\Gamma \cup\left\{\frac{1}{2} \delta_{i}\right\}$ é fechado, pois $\delta_{j}$ possui comprimento menor que n, e então $\Gamma \Vdash \delta_{j}$.

(h2.1): $\Gamma \cup\left\{0\left(\delta_{i} \rightarrow \delta_{n}\right)\right\}$ é fechado, pois $\delta_{j}$ possui comprimento menor que n, e então $\Gamma \Vdash \delta_{j}$. Ainda, se $0\left(\delta_{i} \rightarrow \delta_{n}\right)$ gera um tableau fechado, então temos que:

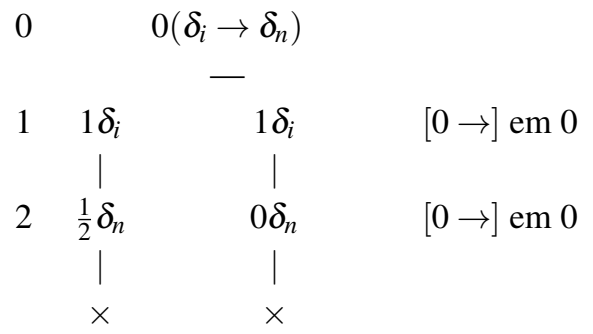

Fechado Hip. Indução

(h2.2): $\Gamma \cup\left\{\frac{1}{2}\left(\delta_{i} \rightarrow \delta_{n}\right)\right\}$ é fechado, pois $\delta_{j}$ possui comprimento menor que n, e então $\Gamma \Vdash \delta_{j}$. Ainda, se $\frac{1}{2}\left(\delta_{i} \rightarrow \delta_{n}\right)$ gera um tableau fechado, então temos que:

$$
\begin{aligned}
& 0 \quad \frac{1}{2}\left(\delta_{i} \rightarrow \delta_{n}\right) \\
& {\left[\frac{1}{2} \rightarrow\right] \text { em } 0}
\end{aligned}
$$

A partir destas informações, extraídas da hipótese de indução, construímos os tableaux fechados para $\Gamma \cup\left\{\neg \delta_{n}\right\}$, por meio das construções seguintes. Iniciamos nosso tableau com $0 \delta_{n}$, em seguida fazemos todas as possíveis suposições de valores para a fórmula $\delta_{i}$. Daí, analisamos todos os casos encontrados.

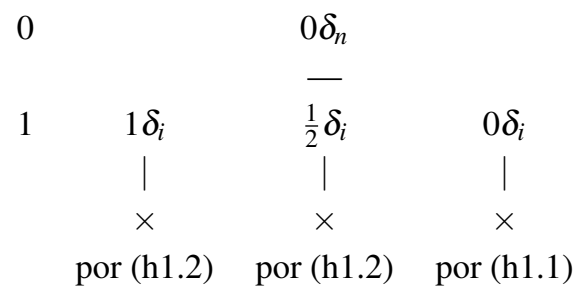


Construiremos agora, o tableau com $\frac{1}{2} \delta_{n}$, em seguida fazemos todas as possíveis suposições de valores para a fórmula $\delta_{i}$. Daí, analisamos todos os casos encontrados.

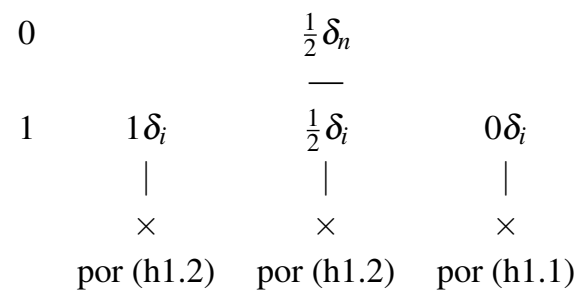

Desta forma, como os axiomas e a regra de inferência de I1 possuem um tableau fechado em TI1, então tudo o que é deduzido em I1 através dos axiomas ou da regra de inferência também possui um tableau fechado em TI1. Logo, pela hipótese de indução aqui mostrada, $\delta_{n}$ é consequência analítica de $\Delta$, ou seja, $\Delta \Vdash \varphi$.

A seguir, demonstraremos a equivalência entre nosso sistema de tableaux TI1 e a consequência semântica da lógica I1.

\subsection{Da dedução em tableaux para a consequência semântica de I1}

Na seção anterior demonstramos que a dedução axiomática da lógica I1 implica na dedução em tableaux de TI1. Para concluirmos a demonstração de equivalência entre a lógica I1 e nosso sistema de tableaux TI1, falta provarmos:

$$
\Gamma \Vdash \varphi \Rightarrow \Gamma \vDash \varphi
$$

Ou seja, que toda dedução analítica pelo nosso sistema de tableaux, tem uma dedução pela consequência semântica da lógica I1. Contudo antes de provarmos este resultado, se faz necessário introduzirmos algumas definições, segundo Silva et. al. [8].

Definição 4.3 (Conjunto descendentemente saturado). Um conjunto $\theta$ de fórmulas marcadas, isto é, fórmulas com valores de verdade $0, \frac{1}{2}$ ou 1 , é dito descendentemente saturado se satisfaz as seguintes condições:

(a) nenhuma fórmula ocorre em $\theta$ com dois valores distintos;

(b) se em $\theta$ ocorre alguma fórmula do tipo $\alpha$, então $\alpha_{1} \in \theta$ e $\alpha_{2} \in \theta$; em que $\alpha_{1}$ e $\alpha_{2}$ denotam as fórmulas decorrentes da aplicação de uma das regras em TII do tipo $\alpha$;

(c) se em $\theta$ ocorre alguma fórmula do tipo $\beta$, então $\beta_{1} \in \theta$ ou $\beta_{2} \in \theta$; em que $\beta_{1}$ e $\beta_{2}$ denotam as fórmulas decorrentes da aplicação de uma das regras em TII do tipo $\beta$;

(d) se em $\theta$ ocorre alguma fórmula do tipo $\gamma$, então $\gamma_{1} \in \theta$ ou $\gamma_{2} \in \theta$ ou $\gamma_{3} \in \theta$. Neste caso, $\gamma_{1}, \gamma_{2}$ e $\gamma_{3}$ denotam as fórmulas decorrentes da aplicação da regra em TII do tipo $\gamma$; 
(e) se em $\theta$ ocorre alguma fórmula do tipo $\delta$, então $\delta_{1} \in \theta$ ou $\delta_{2} \in \theta$ ou $\delta_{3} \in \theta$ ou $\delta_{4} \in \theta$. Aqui, $\delta_{1}, \delta_{2}, \delta_{3}$ e $\delta_{4}$ denotam as fórmulas decorrentes da aplicação de uma das regras em TI1 do tipo $\delta$.

Lema 4.1. Todo ramo saturado e aberto de um tableau é um conjunto descendentemente saturado.

Demonstração. Como o ramo é aberto, então não podem estar presentes no ramo nenhuma fórmula com duas valorações distintas, o que satisfaz a condição (a) da definição de conjunto descendentemente saturado.

Devido a saturação, isto é, como o ramo é completo, segue que todas as possíveis regras do tableau já foram utilizadas e o tableau não pode mais ser expandido.

Logo, se existe uma fórmula do tipo $\alpha$ no ramo, então $\alpha_{1}$ e $\alpha_{2}$ também estão no ramo, o que atende a condição (b).

Pelo mesmo motivo, se há uma fórmula do tipo $\beta$ no ramo, então $\beta_{1}$ ou $\beta_{2}$ está no ramo, o que cumpre a condição (c).

De modo análogo, se ocorre no ramo uma fórmula do tipo $\gamma$ segue que $\gamma_{1}$ ou $\gamma_{2}$ ou $\gamma_{3}$ está no ramo, o que contempla a condição (d). E ainda, do mesmo modo, se ocorre no ramo uma fórmula do tipo $\delta$, segue que $\delta_{1}$ ou $\delta_{2}$ ou $\delta_{3}$ ou $\delta_{4}$ está no ramo, o que contempla a última condição (e). $\square$ Agora, necessitamos estender a noção de valoração para as fórmulas sinalizadas.

Definição 4.4. Se v é uma valoração e $k \in\left\{0, \frac{1}{2}, 1\right\}$, então a fórmula sinalizada $k \varphi$ é distinguida segundo a valoração $v$, o que é denotado por $k \varphi=1$, se $v(\varphi)=k$. Assim, $k \varphi=1 \Leftrightarrow v(\varphi)=k$.

Definição 4.5. Uma valoração $v$ satisfaz um conjunto $\Theta$ de fórmulas sinalizadas se para toda fórmula sinalizada $k \psi$ que ocorre em $\Theta$, tem-se $k \psi=1$.

Definição 4.6. Um conjunto $\Theta$ de fórmulas sinalizadas é satisfatível se existe uma valoração $v$ tal que $v(\Theta)=1$, ou seja, para toda $\psi \in \Theta, k \psi=1$. Assim, se $k \psi \in \Theta$, então $v(\psi)=k$.

Lema 4.2. Se $\Theta$ é um conjunto satisfatível de fórmulas sinalizadas, então:

(i) se uma fórmula do tipo $\alpha$ está em $\Theta$, então $\Theta \cup\left\{\alpha_{1}, \alpha_{2}\right\}$ é satisfatível;

(ii) se uma fórmula do tipo $\beta$ está em $\Theta$, então $\Theta \cup\left\{\beta_{1}\right\}$ é satisfatível ou $\Theta \cup\left\{\beta_{2}\right\}$ é satisfativel;

(iii) se uma fórmula do tipo $\gamma$ está em $\Theta$, então $\Theta \cup\left\{\gamma_{1}\right\}$ é satisfatível, ou $\Theta \cup\left\{\gamma_{2}\right\}$ é satisfatível, ou $\Theta \cup\left\{\gamma_{3}\right\}$ é satisfatível.

(iv) se uma fórmula do tipo $\delta$ está em $\Theta$, então $\Theta \cup\left\{\delta_{1}\right\}$ é satisfatível, ou $\Theta \cup\left\{\delta_{2}\right\}$ é satisfatível, ou $\Theta \cup\left\{\delta_{3}\right\}$ é satisfatível, ou $\Theta \cup\left\{\delta_{4}\right\}$ é satisfatível. 
Demonstração. Analisemos, primeiramente, nossas Regras de Expansão TI1, afim de separar cada regra nos tipos $\alpha, \beta, \gamma$ ou $\delta$. Assim temos:

Regras do tipo $\alpha:[1 \neg]$ e $[1 \wedge]$

Regras do tipo $\beta:[0 \neg],[0 \rightarrow]$ e $[1 \vee]$

Regras do tipo $\gamma:[1 \rightarrow]$

Regras do tipo $\delta:[0 \wedge]$ e $[0 \vee]$

Agora, seguiremos com nossa demonstração, em cada um dos tipos de regras:

(i) Fórmulas do tipo $\alpha$

Tomemos a fórmula de negação do tipo $\alpha$, isto é, $1 \neg A$. Como o conjunto $\Theta$ é satisfatível, então existe uma valoração $v$ tal que $v(\Theta)=1$. Daí, $v(\neg A)=1$ e então $v(A)=0$, portanto $v(\Theta \cup\{0 A\})=1$.

Agora a conjunção do tipo $\alpha$, isto é, $1 A \wedge B$. Como o conjunto $\Theta$ é satisfatível, então existe uma valoração $v$ tal que $v(\Theta)=1$. Daí, $v(A \wedge B)=1$, então $v(A)=1$ e $v(B)=1$. Portanto, $v(\Theta \cup\{1 A, 1 B\})=1$.

(ii) Fórmulas do tipo $\beta$

Para a fórmula de negação do tipo $\beta$, temos $0 \neg A$. Como o conjunto $\Theta$ é satisfatível, então existe uma valoração $v$ tal que $v(\Theta)=1, \operatorname{logo}, v(\neg A)=0$. Portanto, $v(A)=1$ ou $v(A)=\frac{1}{2}$. Se $v(A)=1$, então $v(\Theta \cup\{1 A\})=1$. Contudo, se $v(A)=\frac{1}{2}$, então $v\left(\Theta \cup\left\{\frac{1}{2} A\right\}\right)=1$. De qualquer modo há um ramo tal que $v(\Theta \cup\{k A\})=1$.

Agora a condicional do tipo $\beta$, temos $0(A \rightarrow B)$. Como o conjunto $\Theta$ é satisfatível, então existe uma valoração $v$ tal que $v(\Theta)=1, \operatorname{logo}, v(A \rightarrow B)=0$. Portanto $v(A)=1$ e $v(B)=\frac{1}{2}$; ou $v(A)=1$ e $v(B)=0$. Se $v(A)=1$ e $v(B)=\frac{1}{2}$, então $v\left(\Theta \cup\left\{1 A, \frac{1}{2} B\right\}\right)=1$. Contudo, se $v(A)=1$ e $v(B)=0$, então $v(\Theta \cup\{1 A, 0 B\})=1$. De qualquer modo há um ramo tal que $v(\Theta \cup\{\{1 A, k B\}\})=1$.

Agora a disjunção do tipo $\beta$, temos $1(A \vee B)$. Como o conjunto $\Theta$ é satisfatível, então existe uma valoração $v$ tal que $v(\Theta)=1, \operatorname{logo}, v(A \vee B)=1$. Portanto $v(A)=1$ ou $v(B)=1$. Se $v(A)=1$, então $v(\Theta \cup\{1 A\})=1$. Contudo, se $v(B)=1$, então $v(\Theta \cup\{1 B\})=1$.

(iii) Fórmulas do tipo $\gamma$

Para a fórmula do condicional do tipo $\gamma$, temos $1(A \rightarrow B)$. Como o conjunto $\Theta$ é satisfatível, então existe uma valoração $v$ tal que $v(\Theta)=1$, $\log o, v(A \rightarrow B)=1$. Portanto $v(A)=0$; ou $v(A)=\frac{1}{2}$; ou $v(B)=1$. Se $v(A)=0$, então $v(\Theta \cup\{0 A\})=1$, contudo, se $v(A)=0$, então $v\left(\Theta \cup\left\{\frac{1}{2} A\right\}\right)=1$, ou ainda se, $v(B)=1$, então $v(\Theta \cup\{1 B\})=1$. Logo, podemos concluir que, seja $k \in\left\{0, \frac{1}{2}\right\}$, se $v(A)=k$, então $v(\Theta \cup\{k A\})=1$, e se $v(B)=1$, então $v(\Theta \cup\{1 B\})=1$. 
(iv) Fórmulas do tipo $\delta$

Para a fórmula da conjunção do tipo $\delta$, temos $0(A \wedge B)$. Como o conjunto $\Theta$ é satisfatível, então existe uma valoração $v$ tal que $v(\Theta)=1$. Daí, $v(A \wedge B)=0$, então $v(A)=0$; ou $v(A)=\frac{1}{2}$; ou $v(B)=0$; ou $v(B)=\frac{1}{2}$. Como são quatro casos, temos que, se (caso a) $v(A)=0$, então $v(\Theta \cup\{0 A\})=1$. Contudo se (caso b), $v(A)=\frac{1}{2}$, então $v\left(\Theta \cup\left\{\frac{1}{2} A\right\}\right)=1$. E no caso em que (caso c) se $v(B)=0$, então $v(\Theta \cup\{0 B\})=1$. E ainda (caso d) se $v(B)=\frac{1}{2}$, então $v\left(\Theta \cup\left\{\frac{1}{2} B\right\}\right)=1$. Podemos ainda generalizar, seja $k \in\left\{0, \frac{1}{2}\right\}$. Se $v(A)=k$, então $v(\Theta \cup\{k A\})=1$, e se $v(B)=k$, então $v(\Theta \cup\{k B\})=1$.

Para a fórmula da disjunção do tipo $\delta$, temos $0(A \vee B)$. Como o conjunto $\Theta$ é satisfatível, então existe uma valoração $v$ tal que $v(\Theta)=1, \log o, v(A \vee B)=0$. Portanto $v(A)=0$ e $v(B)=\frac{1}{2}$; ou $v(A)=\frac{1}{2}$ e $v(B)=\frac{1}{2} ; v(A)=0$ e $v(B)=0$; ou $v(A)=\frac{1}{2}$ e $v(B)=0$. Portanto, novamente temos aqui quatro casos, (caso a) se $v(A)=0$ e $v(B)=\frac{1}{2}$, então $v(\Theta \cup$ $\left.\left\{0 A, \frac{1}{2} B\right\}\right)=1$. No caso em que (caso b) se $v(A)=\frac{1}{2}$ e $v(B)=\frac{1}{2}$, então $v\left(\Theta \cup\left\{\frac{1}{2} A, \frac{1}{2} B\right\}\right)=$ 1. No caso em que (caso c) se $v(A)=0$ e $v(B)=0$, então $v(\Theta \cup\{0 A, 0 B\})=1$. E ainda no caso em que (caso d) se $v(A)=\frac{1}{2}$ e $v(B)=0$, então $v\left(\Theta \cup\left\{\frac{1}{2} A, 0 B\right\}\right)=1$. Podemos ainda generalizar da seguinte forma. Seja $k_{1} \in\left\{0, \frac{1}{2}\right\}$, e $k_{2} \in\left\{0, \frac{1}{2}\right\}$. Daí, $v(A)=k_{1}$ e $v(B)=k_{2}$, portanto $v\left(\Theta \cup\left\{k_{1} A, k_{2} B\right\}\right)=1$.

Sendo assim, em todos os casos, analisando todas a regras de expansão de nosso sistema TI1, algum ramo do tableau é satisfatível.

Diante dessas definições e do lema acima, podemos agora então provar o seguinte teorema:

Teorema 4.2. Se $\Gamma \Vdash \varphi$, então $\Gamma \vDash \varphi$

Demonstração. Realizaremos esta demonstração, pela contra-positiva, isto é, assumir que $\Gamma$ não deduz semanticamente $\varphi$ e provar que $\Gamma$ não gera um tableau fechado para $\varphi$.

De fato, suponha que $\Gamma$ não deduz semanticamente $\varphi$. Então, existe uma valoração de $v$ tal que $v(\Gamma)=1$ e $v(\varphi)=k$, sendo $k \in\left\{0, \frac{1}{2}\right\}$.

Seja $\Theta_{0}$ o conjunto de fórmulas marcadas pelos valores de $v$ em fórmulas de $\Gamma$, i.e., que ocorrem no tableau inicial. Por construção, $v\left(\Theta_{0}\right)=1$. Provaremos que a cada passo da expansão do tableau, haverá sempre um ramo $\Theta_{i}$ tal que $v\left(\Theta_{i}\right)=1$.

Suponha que $v\left(\Theta_{i-1}\right)=1$. Se o ramo $\Theta_{i-1}$ for expandido por uma fórmula do tipo $\alpha$, pelo Lema anterior, item (i), temos que $v\left(\Theta_{i}\right)=1$.

No caso do ramo $\Theta_{i-1}$ for expandido por uma fórmula do tipo $\beta$, segue pelo Lema anterior, item (ii), que $v\left(\Theta_{i}\right)=1$.

Se o ramo $\Theta_{i-1}$ for expandido por uma fórmula do tipo $\gamma$, segue pelo mesmo Lema, item (iii), que $v\left(\Theta_{i}\right)=1$. 
E ainda, se o ramo $\Theta_{i-1}$ for expandido por uma fórmula do tipo $\delta$, segue pelo mesmo Lema, item (iv), que $v\left(\Theta_{i}\right)=1$.

Assim, em todos os casos, temos um ramo $\Theta_{i}$ tal que $v\left(\Theta_{i}\right)=1$. Logo, sempre haverá um ramo satisfatível em $\Theta$, que após todas as expansões, será um conjunto descendentemente saturado, que não se pode fechar. Portanto, $\Gamma$ não gera um tableau fechado para $\varphi$.

Portanto, provamos que $\Gamma \Vdash \varphi \Rightarrow \Gamma \vDash \varphi$

Desta forma, pelos Teoremas 4.1 e 4.2 estabelecemos as etapas 1 e 2 da demonstração da equivalência entre os sistemas I1 e TI1.

\title{
5 CONSIDERAÇÕES FINAIS
}

Neste artigo, apresentamos a lógica trivalente e intuicionista I1, originalmente introduzida por Sette e Carnielli [7]. Após o estudo detalhado da sintaxe e semântica da lógica I1, desenvolvemos um sistema de tableaux chamado TI1, em que sua equivalência dedutiva com o sistema axiomático I1 foi demonstrada no presente artigo, por meio dos Teoremas 4.1 e 4.2.

Ademais, destacamos as vantagens de nosso sistema de tableaux, TI1, se apresentar como uma árvore ordenada quadriádica, tornando este mais aplicável do ponto de vista computacional, por ser um método, por vezes, mais prático e rápido, pois constitui-se num sistema de prova automática de teoremas, enquanto em I1 falta localidade quando da escolha de axiomas e sua respectiva instanciação na construção de uma dedução.

\section{AGRADECIMENTOS}

Agradecemos ao Conselho Nacional de Desenvolvimento Científico e Tecnológico (CNPq) pelo fomento a esta pesquisa.

\begin{abstract}
Sette and Carnielli introduced the axiomatic system I1 in 1995, which presents an intuitionistic character, in the same regard of the logical system developed by Arend Heyting (1898-1980), that is, as a logic underlying to the constructive mathematics, for instance, $\neg \neg A \rightarrow A$ is not an I1 tautology. On the other hand, this system is a 3-valued logic, in which presents in addition to the classical truth values, the truth-value $\mathrm{F}^{*}$, that can be understood as "false by lack of positive evidence". In the semantic environment of this logic, there is only one distinguished value. In this work, we develop an alternative deductive method to the axiomatic for the system I1, namely, we introduce an analytic tableaux system for such logic. We establish, by means of a theorem, that every deduction obtained from the axiomatic system, will be also deduced by the tableaux system proposed.
\end{abstract}

Keywords: intuitionistic logic, three-valued logic, method of analytic tableaux. 


\section{REFERÊNCIAS}

[1] W.A. Carnielli \& M. Lima-Marques. Society semantics for multiple-valued logics. In W. Carnielli \& I. D’Ottaviano (editors), "Advances in Contemporary Logic and Computer Science”, volume 235 of Contemporary Mathematics Series. American Mathematical Society (1999), p. 33-52.

[2] G. da S. Queiroz. "Sobre a Dualidade entre Intuicionismo e Paraconsistência". Ph.D. thesis, IFCH Universidade Estadual de Campinas, Campinas (1998).

[3] V.L. Fernández \& M.E. Coniglio. Combining Valuations with Society Semantics. Journal of Applied Non-Classical Logics, Paris, 13 (2003), 21-46.

[4] M.C. Fitting. Introduction. In M. D’Agostino, D.V. Gabbay, R. Hahnle \& J.Posegga (editors), "Handbook of Tableaux Methods". Kluwer Academic Publishers (1999), p. 1-43.

[5] A. Loparic \& N.C.A. da Costa A. Paraconsistency, paracompleteness and valuations. Logique et Analyse, 106 (1984), 119-131.

[6] C.A. Mortari. "Introdução à Lógica”. Editora Unesp, São Paulo (2001).

[7] A.M. Sette \& W.A. Carnielli. Maximal Weakly-intuicionistic logics. Studia Logica, 55 (1995), 181203.

[8] F.S.C. Silva, M. Finger \& A.C.V. Melo. "Lógica para computação". Thomson Learning, São Paulo (2006).

[9] L.H.C. Silvestrini. Cálculo trivalorado, quase verdade e sociedades paraconsistentes (2018). URL https://proceedings.sbmac.org.br/sbmac/article/view/2552/2571. Acesso em: 15 dez. 2018.

[10] R.M. Smullyan. "First-order logic". Dover Publication, New York (1968). 